\title{
Grupos recurrentes de peces y crustáceos demersales en la zona centro-sur de Chile
}

\author{
Brunilda Menares \& José I. Sepúlveda \\ Escuela de Ciencias del Mar, Pontificia Universidad Católica de Valparaíso \\ Casilla 1020, Valparaíso, Chile
}

\begin{abstract}
RESUMEN. Se estudia la estructura comunitaria de peces y crustáceos demersales, su relación con las condiciones hidrográficas, y el tipo de sustrato en áreas de pesca de Merluccius gayi, en la zona centro-sur de Chile $\left(31^{\circ}\right.$ a $\left.41^{\circ} \mathrm{S}\right)$ entre el 25 de julio y el 1 de septiembre de 1993. Con este propósito se calcularon los índices de diversidad (ShannonWeaver), Similitud (Sanders), Grupos Recurrentes (Fager y McGowan), Importancia Relativa (Fager), y se realizó el Análisis de Correlación Canónica. El área de estudio presentó una similitud faunística constituida por 35 especies comunes, de las cuales Merluccius gayi e Hippoglossina macrops fueron las especies dominantes del sistema. Se reconoce tres asociaciones de fauna íctica y de crustáceos bentodemersales. Una se distribuye entre el límite de la plataforma continental y la zona batial; otra, restringida al límite de la plataforma continental y la tercera, en la zona del talud superior. Las asociaciones de los grupos recurrentes se encuentran asociadas a las Aguas Ecuatoriales Subsuperficiales y a la mezcla entre el Agua Ecuatorial Subsuperficial y el Agua Intermedia Antártica.
\end{abstract}

Palabras clave: fauna bentodemersal, grupos recurrentes, hidrografía, sustrato,Chile.

\section{Recurrent groups of demersal fishes and crustaceans of the south-central zone off Chile}

\begin{abstract}
The community structure of demersal fishes and crustaceans and its' relationship to hydrographic and bottom features was evaluated based on data from bio-oceanographic cruises made off the S-central Chilean coast between $31^{\circ}$ and $41^{\circ} \mathrm{S}$ from 25 July to 01 September 1993. The Shannon-Weaver diversity index, Sanders' similarity index, Fager's ranking procedures, Fager \& McGowan recurrent group analysis, and Canonical Correlation Analysis were used for interpretations of the data. The area studied showed a faunal similarity composed of 35 species in common, with Merluccius gayi and Hippoglossina macrops as dominant species in the system. Three associations of fishes and crustaceans were identified, including one distributed between the upper limit of the continental shelf and the bathyal zone, a second was limited to the edge of the continental shelf, and the third to the upper region of the slope. The recurrent groups were associated with Subsurface Equatorial Water and Antarctic Intermediate Water.
\end{abstract}

Key words: demersal fauna, recurrent groups, hydrography, substrate, Chile.

\section{INTRODUCCIÓN}

La organización de asociaciones de peces está determinada en diferentes áreas geográficas principalmente por la profundidad y la estructura hidrográfica. Sin embargo, es posible diferenciar patrones que van desde zonaciones faunísticas con

Autor corresponsal: José I. Sepúlveda (jisv@ yahoo.com) bordes espaciales marcados hasta cambios graduales que no muestran discontinuidades espaciales (Fariña et al., 1997; Jacob et al., 1998).

La variabilidad en las condiciones ambientales serían factores importantes que contribuyen a la tendencia observada en la composición y abundancia de la comunidad (Rocha \& Rossi-Wongtschowski, 1998). Factores tales como temperatura, sustrato, 
productividad y requerimientos biológicos, influyen potencialmente en la distribución del taxa y fundamentalmente en la distribución de asociaciones de especies (Jacob et al., 1998; Fariña et al., 1997).

La plataforma y la parte superior del talud continental son áreas donde ocurren los mayores cambios en la fauna, principalmente a lo largo del gradiente batimétrico (Overholtz \& Tyler, 1985; Fariña et al., 1997). Así, al aumentar la profundidad se observaría un decrecimiento de la densidad, la biomasa y menor riqueza de especies de peces demersales (Fariña et al., 1997). Sin embargo, dentro de cada estrato de agua otros factores tales como temperatura, salinidad, contenido de oxígeno, sistemas de corrientes, naturaleza del sustrato, así como cambios en la biota se convierten en factores relevantes en la composición de la comunidad (Jacob et al., 1998; Rocha \& Rossi-Wongstschowski, 1998).

Arana et al. (1975) y Yáñez (1978), señalan que la fauna asociada a las capturas de arrastre realizadas en la región de Valparaíso, está compuesta por un número apreciable de individuos y familias caracterizados principalmente por las clases Chondrichthyes, Osteichthyes, Crustacea, Myxini, Elasmobranchii, Holocephali y Teleostomi. Arancibia (1992), también encontró que la fauna de la plataforma continental (1-200 m) y aquella de la zona batial (200-600 m) presentan una notable discontinuidad. Al interior de estas dos zonas la composición faunística varía gradualmente con la profundidad. Así, la fauna que forma parte de la plataforma y la del talud continental aparecerían conectadas entre sí por algunas especies invasoras, de una u otra zona, las cuales no presentan rangos restringidos de distribución en profundidad. La diferenciación gradual de estas dos comunidades estaría reflejada por aquellas familias representadas en ambos grupos por especies diferentes y que probablemente ocupan nichos ecológicos similares.

El progresivo ensanchamiento de la plataforma continental en sentido norte sur a lo largo del litoral chileno ha permitido el desarrollo de una importante pesquería de peces demersales desde Coquimbo hacia al sur, donde se han diferenciado dos zonas pesqueras que coinciden con los rangos de distribución latitudinal de la merluza común (M. gayi), que se extiende hacia el sur hasta alrededor de los $43^{\circ} \mathrm{S}$, y $M$. australis que aparece desde los $37^{\circ}$ hasta los $57^{\circ} \mathrm{S}$, pero distribuyéndose principalmente entre los $41^{\circ} 28^{\prime}$ y $57^{\circ} \mathrm{S}$ (Ojeda, 1983; Céspedes et al., 2001). M. gayi se distribuye desde Antofagasta $\left(23^{\circ} 38^{\prime}\right.$ 'S) hasta el Canal Cheap (470'S) (Palma et al., 1998), siendo explotada principalmente entre Los Vilos (31 $55^{\circ}$ 'S) y Corral (39 $59^{\circ}$ 'S). Los cruceros de evaluación de $M$. gayi realizados por el Instituto de Fomento Pesquero en la zona centro-sur de Chile han detectado que esta pesquería está constituida por otras especies acompañantes como el lenguado de ojos grandes (Hippoglossina macrops), congrio negro (Genypterus maculatus) y langostino colorado (Pleuroncodes monodon) a lo largo de la plataforma continental y talud superior de Chile central ( $30^{\circ}$ $39^{\circ} \mathrm{S}$ ) (Arancibia, 1991, 1992).

La distribución de merluza común está asociada al Agua Ecuatorial Subsuperficial (AESS), habitando la plataforma y el talud continental entre 20 y $550 \mathrm{~m}$ de profundidad. Su distribución batimétrica está relacionada a desplazamientos estacionales y a migraciones nictimerales que corresponderían a un comportamiento trófico (Aguayo \& Robotham, 1984; Cañón \& Morales, 1985; Vidal et al., 1985; Zamora, 1991; Palma et al., 1998).

En general, las investigaciones tanto de la fauna íctica como de la fauna de crustáceos frente a Chile central son el resultado de cruceros y expediciones dirigidas a la evaluación específica de determinados recursos pesqueros más que al análisis de comunidades y su relación con las características oceanográficas. En este trabajo se pretende relacionar la captura para un período de invierno ( 25 de julio al 1 de septiembre de 1993), en cuanto a su composición específica, con las características del hábitat donde fueron encontrados (profundidad, temperatura, salinidad, oxígeno disuelto, masas de aguas y tipo de sustrato), en un intento por describir la estructura comunitaria en la zona centro-sur de Chile.

\section{MATERIALES Y MÉTODOS}

Durante el crucero de investigación pesquera, realizado entre el 25 de julio y el 1 de septiembre de 1993, se efectuaron 133 lances de pesca (estaciones), entre $31^{\circ} 00$ y $41^{\circ} 28^{\prime} \mathrm{S}$ (Fig. 1), en los que se colectaron muestras de la fauna de peces y crustáceos mediante pescas de arrastre con redes de fondo merluceras. La identificación y cuantificación de la fauna se realizó a bordo de la embarcación utilizando las claves taxonómicas de Wisner (1974) para los peces y de Burukovskii (1992); Retamal (1981, 1994) y Pohle et al. (1999), para los crustáceos. 


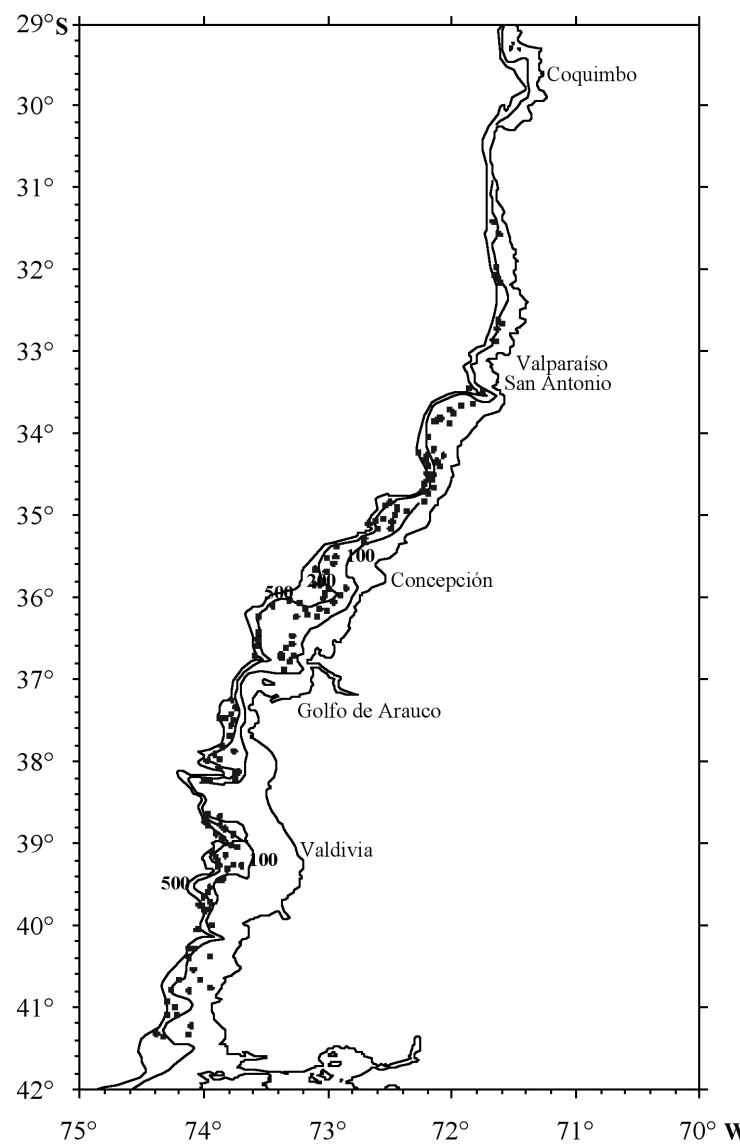

Figura 1. Posición geográfica de las estaciones de pesca del crucero biológico-oceanográfico, realizado entre $31^{\circ}$ y $41^{\circ} \mathrm{S}$ (julio-septiembre, 1993).

Figure 1. Geographic location of fishing stations of the biological oceanographic cruise conducted between $31^{\circ}$ and $4^{\circ} \mathrm{S}$ (July-September, 1993).

Simultáneamente, se efectuaron 80 estaciones oceanográficas distribuidas en 24 transectas perpendiculares a la costa. En cada estación se registró el perfil de temperatura, salinidad y oxígeno disuelto en la columna de agua, con un CTD Neil BrownMark III. Paralelamente a las estaciones de muestreo, durante la noche, se hicieron barridos acústicos con ecosonda Simrad EK500 para determinar el tipo de fondo, abarcando la plataforma desde la costa hasta la isóbata de $500 \mathrm{~m}$. Con los resultados obtenidos de tales mediciones y la información de los fondos proveniente de cruceros de pescas exploratorias efectuados en la misma área de estudio $\left(29^{\circ}-41^{\circ} \mathrm{S}\right)$, se generó una base de datos de los tipos de fondo de la zona de estudio, utilizando la nomenclatura de la descripción del tipo de fondo de acuerdo con las bitácoras de pesca de cada crucero. Como complemento se utilizó las cartas pesqueras CP-400, CP600 y CP-700 (SHOA, 1981, 1983, 1984).

La zona de trabajo fue dividida, según la clasificación del ambiente marino sugerida por Lalli \& Parsons (1993), en cuatro intervalos de profundidad:

$$
\begin{array}{lr}
\text { Sublitoral exterior } & 51-100 \mathrm{~m} \\
\text { Límite de la plataforma } & 101-200 \mathrm{~m} \\
\text { Talud superior } & 201-350 \mathrm{~m} \\
\text { Zona batial } & 351-550 \mathrm{~m}
\end{array}
$$

Para la evaluación de la comunidad en términos de diversidad, abundancia, similitud y asociación no se consideran los lances al sur de los $38^{\circ} \mathrm{S}$ debido a que únicamente se capturó merluza común. Los índices utilizados fueron los siguientes:

La diversidad fue calculada utilizando el índice de Shannon \& Weaver (1949), cuyos resultados se expresan en bits.ind. ${ }^{-1}$ (Pielou, 1977). Se consideraron núcleos de alta diversidad aquellas zonas con valores iguales o superiores a dos bits ( 2,0 bits) y núcleos de baja diversidad a aquellas zonas con valores menores a dos bits $(<2,0$ bits $)$.

La similitud faunística se determinó utilizando el índice de Sanders (1960), el cual compara la abundancia relativa de cada especie, expresada como porcentaje de la captura total por lance para cada especie, en todos los pares posibles de muestras. El menor porcentaje de los dos se toma como un valor de abundancia común. Estos valores son sumados y la suma representa una medida del porcentaje de la fauna común de ambas muestras.

Con el fin de establecer la importancia relativa de las especies dominantes se utilizó el ordenamiento de Fager (1957), donde las especies se ordenan de 1 a 10 por abundancia dentro de cada muestra. A la especie más abundante se le asigna el valor 10, a la siguiente 9 , y así sucesivamente, hasta llegar al número de orden 10 al que se le asigna el valor 1 . Se suman los números de orden sobre los lances considerados y se divide por el número de lances.

Para determinar las asociaciones de especies en el período de estudio se utilizó el índice de Fager \& McGowan (1963), según la siguiente expresión:

$$
I F M=\frac{J}{\sqrt{N_{a} \cdot N_{b}}}-\frac{1}{2 \sqrt{N_{b}}}
$$


donde:

$\mathrm{J}=$ número de ocurrencias conjuntas

$\mathrm{N}_{\mathrm{a}}=$ número de ocurrencia de la especie a (es el menor número de ocurrencia)

$\mathrm{N}_{\mathrm{b}}=$ número de ocurrencia de la especie $\mathrm{b}$

Se considera que las especies presentan afinidad cuando los valores de esta expresión son iguales o mayores que 0,5 . El grado de asociación entre los grupos recurrentes fue estimado como la razón del número de afinidades de pares de especies por sobre el nivel 0,5 con respecto al máximo número de afinidades posibles (Fager, 1957).

Para establecer el grado de interrelación entre la abundancia relativa de las especies y las variables abióticas (profundidad, temperatura, salinidad, concentración de oxígeno disuelto, densidad y sustrato), se utilizó el Análisis de Correlación Canónica (ACC), el cual se basa en la obtención de la máxima correlación posible entre dos conjuntos de variables (Manly, 1994). El análisis se realizó utilizando la abundancia relativa normalizada de las especies registradas y que estuvieron presentes a lo menos en un 20\% del total de los lances. Finalmente, se aplicó el ACC para los grupos recurrentes, empleando la abundancia relativa normalizada de las especies que componen cada grupo recurrente. Estos cálculos se efectuaron mediante el programa computacional Statistica 5.1.

\section{RESULTADOS}

\section{Naturaleza del sustrato}

La zona de estudio se caracterizó por la presencia de cuatro tipos de sustratos: roca-laja, arena, fangoarena-roca y fango. El área comprendida entre $29^{\circ}$ y $31^{\circ} \mathrm{S}$ está constituida por tres tipos de sustratos, fango-arena-roca, arena y roca-laja. Los dos primeros sustratos están presentes en el sector de la plataforma en forma de pequeños núcleos, en cambio la roca-laja corresponde al sustrato predominante, que se distribuye desde la plataforma a la zona batial. Entre $31^{\circ}$ y $38^{\circ} \mathrm{S}$, el sustrato dominante es la asociación fango-arena-roca, el cual se ubica hasta los 500 $\mathrm{m}$. Tanto latitudinal como batimétricamente existen núcleos de fango, arena y roca-laja, en forma intermitente.

\section{Condiciones hidrográficas}

La mayor parte de los lances de pesca se realizaron en las cercanías del fondo (estratos 101-200 y 201$350 \mathrm{~m}$ de profundidad), donde las características ambientales de la zona de estudio indican la presencia de AESS, con concentraciones de oxígeno inferiores a $3 \mathrm{~mL} \cdot \mathrm{L}^{-1}$, temperaturas entre $7^{\circ}$ y $12^{\circ} \mathrm{C}$ y salinidades entre 34,40 y 34,80 psu. Entre $31^{\circ}$ y $37^{\circ} \mathrm{S}$ la temperatura de fondo en la plataforma continental fluctuó entre $11^{\circ}$ y $12^{\circ} \mathrm{C}$; en el talud superior la temperatura fue más baja, fluctuando entre $8^{\circ}$ y $10^{\circ} \mathrm{C}$, mientras que en la zona batial las aguas fueron más frías $\left(<8^{\circ} \mathrm{C}\right)$. Al sur de los $37^{\circ} \mathrm{S}$ se observó un descenso de la temperatura desde la plataforma a la zona batial, así en la plataforma las temperaturas oscilaron entre $9^{\circ}$ y $10^{\circ} \mathrm{C}$, mientras que en el estrato 201 $350 \mathrm{~m}$, las temperaturas registraron valores menores a $8^{\circ} \mathrm{C}$ y en la zona batial las temperaturas fueron aún más frías $\left(<6^{\circ} \mathrm{C}\right)$. Entre $31^{\circ}$ y $37^{\circ} \mathrm{S}$, en la plataforma, la salinidad de fondo presentó valores superiores a $34,6 \mathrm{psu}$. Entre $37^{\circ}$ y $38^{\circ} \mathrm{S}$ en este mismo sector, la salinidad disminuyó a 34,5 psu. Desde el talud superior hasta la zona batial, la salinidad varió entre 34,4 y $34,7 \mathrm{psu}$. Al sur de los $38^{\circ} \mathrm{S}$ se apreció un descenso de la salinidad con valores que fluctuaron entre 34,4 y 34,3 psu desde la plataforma hasta la zona batial. Entre $31^{\circ}$ y $41^{\circ} \mathrm{S}$ la densidad (sigma-t) de fondo varió de 26,4 a 26,5 en la plataforma continental, pero entre $37^{\circ}$ y $38^{\circ} \mathrm{S}$ se observó un leve aumento de la densidad (26,7). En cambio en los estratos más profundos, desde el talud superior a la zona batial, la densidad fluctuó entre 26,4 y 26,7 , llegando en algunos sectores a 27,0 . Entre $31^{\circ}$ y $37^{\circ} \mathrm{S}$ el oxígeno disuelto en el fondo desde la plataforma a la zona batial presentó concentraciones inferiores a $1,5 \mathrm{~mL} \cdot \mathrm{L}^{-1}$. Sin embargo, a partir de los $36^{\circ} \mathrm{S}$ desde el sector del talud superior hasta la zona batial, la concentración de oxígeno fue superior a $2,0 \mathrm{~mL} \cdot \mathrm{L}^{-1}$. Entre $37^{\circ}$ a $38^{\circ} \mathrm{S}$, el oxígeno disuelto comenzó aumentar en todos los estratos batimétricos, con valores superiores a $2,5 \mathrm{~mL} \cdot \mathrm{L}^{-1}$.

\section{Composición faunística}

Los núcleos de alta diversidad se registraron entre $33^{\circ}$ y $34^{\circ} \mathrm{S}$, localizándose batimétricamente en el límite de la plataforma. Un segundo foco de alta diversidad se encontró en los $36^{\circ} \mathrm{S}$, desde el límite del talud superior hasta la zona batial. Otro núcleo de alta diversidad se detectó en los $38^{\circ} \mathrm{S}$, desde el límite de la plataforma hasta la zona batial. En los núcleos de alta diversidad (>2,0 bits), el $90 \%$ de la 
composición faunística estuvo integrada por las especies: Coelorhynchus aconcagua, Libidoclaea granaria, Merluccius gayi, Mursia gaudichaudi, Cervimunida johni, Hippoglossina macrops, Cancer porteri y Centroscyllium nigrum. En el resto del área (diversidad $<2,0$ bits), fue representada por C. johni, Epigonus crassicaudus, L. granaria, M. gayi y Pleuroncodes monodon.

El índice de similitud de Sanders se aplicó para un total de 96 estaciones, 64 de ellas resultaron ser similares en un $80 \%$ en su abundancia relativa. En la estructura interna de dichas estaciones se observó que la composición de especies estuvo integrada por 35 especies (Tabla 1).

Según el ordenamiento de Fager, de un total de 96 lances y 38 especies presentes se determinaron las 10 especies más importantes: $M$. gayi fue la especie dominante seguida por $H$. macrops, $L$. granaria, C. porteri, M. gaudichaudi, $C$. aconcagua, $P$. monodon, $R$. chilensis, $G$. maculatus y E. crassicaudus.

\section{Asociación de especies}

De un total de 38 especies presentes entre $31^{\circ}$ y $38^{\circ} \mathrm{S}, 15$ de ellas se asociaron conformando tres grupos; otras dos especies presentaron afinidades con los miembros de uno de los grupos; dos especies estuvieron solitarias y no se asociaron a ninguno de los tres grupos; y 19 especies no mostraron afinidades con ninguno de los grupos formados (Fig. 2). El grupo recurrente A (GRA), estuvo integrado por seis especies $(M$. gayi, R. chilensis, G. blacodes, E. crassicaudus, L. granaria y $C$. aconcagua) y dos especies asociadas. El GRA está interconectado con el grupo recurrente B (GRB), constituido por $H$. macrops, $H$. canescens, $C$. nigrum, $M$. gaudichaudi y $C$. porteri y el grupo recurrente C (GRC), fue formado por Bathyraja sp., M. magellanicus, G. maculatus y S. oculatus (Fig. 2). Los GRA y GRB presentaron un valor de
Tabla 1. Composición de especies en los grupos de estaciones formados al aplicar el índice de Sanders entre $31^{\circ}$ y $38^{\circ} \mathrm{S}$ (julio-septiembre, 1993).

Table 1. Species composition of stations groupings formed by Sander's index between $31^{\circ}$ and $38^{\circ} \mathrm{S}$ (July-September, 1993).

\begin{tabular}{|c|c|}
\hline \multicolumn{2}{|c|}{ PECES } \\
\hline Squalidae & Merlucciidae \\
\hline Centroscylliun nigrum & Merluccius gayi \\
\hline Centroscymnus crepidater & Macrouridae \\
\hline Deania calcea & Coelorhynchus aconcagua \\
\hline Squalus acanthias & Coelorhynchus fasciatus \\
\hline Scyliorhinidae & Ophidiidae \\
\hline Apristurus nasatus & Genypterus chilensis \\
\hline Halaelurus canescens & Genypterus blacodes \\
\hline Triakidae & Genypterus maculatus \\
\hline Mustelus mento & Berycidae \\
\hline Rajidae & Beryx splendens \\
\hline Bathyraja sp. & Scorpenidae \\
\hline Psammobatis sp. & Sebastes oculatus \\
\hline Raja chilensis & Apogonidae \\
\hline Raja sp. & Epigonus crassicaudus \\
\hline Torpedinidae & Branchiostegidae \\
\hline Torpedo tremens & Prolatilus jugularis \\
\hline Chimaeridae & Carangidae \\
\hline Hydrolagus sp. & Trachurus murphyi \\
\hline Congridae & Bramidae \\
\hline Bassanago albescens & Bramma australis \\
\hline Gadidae & Bothidae \\
\hline Macruronus magellanicus & Hippoglossina macrops \\
\hline \multicolumn{2}{|c|}{ CRUSTÁCEOS } \\
\hline Majidae & Pandalidae \\
\hline Libidoclaea granaria & Heterocarpus reedi \\
\hline Cancridae & Galatheidae \\
\hline Cancer porteri & Cervimunida johni \\
\hline Calappidae & Pleuroncodes monodon \\
\hline \multirow[t]{2}{*}{ Mursia gaudichaudi } & Squillidae \\
\hline & Pterygosquilla armata \\
\hline
\end{tabular}

afinidad intergrupo de 0,40. En cambio el valor de afinidad para GRA y GRC fue 0,29 y el valor de afinidad intergrupo para los GRB y GRC fue de 0,10 (Fig. 2). Tres rangos batimétricos delimitaron la distribución de las especies bentodemersales: 51-100, $150-200$ y $250-350 \mathrm{~m}$. 


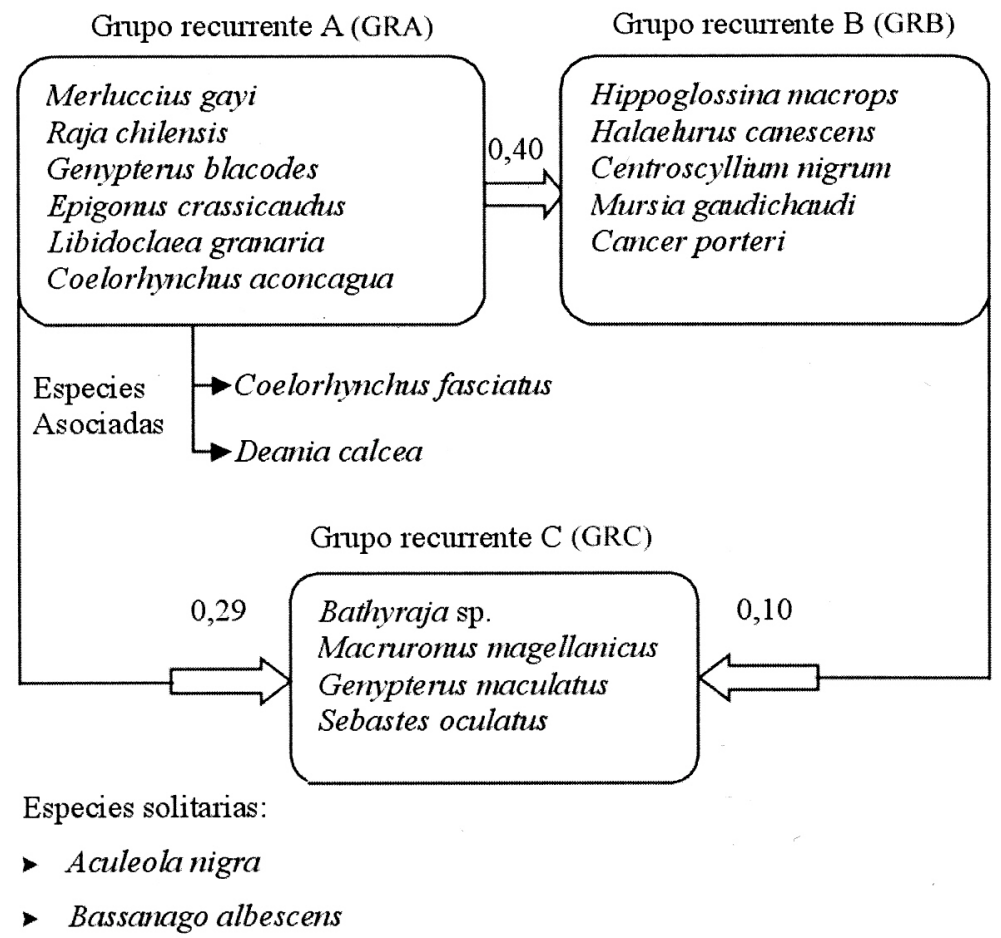

Figura 2. Afinidades intergrupo de especies recurrentes, entre $31^{\circ}$ y $38^{\circ} \mathrm{S}$ (julio-septiembre 1993). GRA: Grupo recurrente A; GRB: Grupo recurrente B; GRC: Grupo recurrente C.

Figure 2. Intergroup affinities of species recurrent groups, between $31^{\circ}$ and $38^{\circ} \mathrm{S}$ (July-September, 1993). GRA: Recurrent group A, GRB: Recurrent group B, GRC: Recurrent group C.

\section{Análisis de Correlación Canónica (ACC)}

Del ACC resultó un índice global de correlación canónica de $\mathrm{R}=0,798$, siendo significativos $(\mathrm{p}<0,1)$ los dos primeros pares de vectores, con varianza explicada de 37,618 y $24,823 \%$, respectivamente (Tabla 2). El primer par de variables canónicas expresó en mayor grado a las especies $L$. granaria, $E$. crassicaudus, C. aconcagua, $H$. canescens, $G$. blacodes en forma positiva y a $M$. gaudichaudi negativamente, y a las variables abióticas profundidad y densidad positivamente, mientras que negativamente a temperatura y salinidad. Lo anterior, indicaría que la abundancia relativa de $L$. granaria, E. crassicaudus, $C$. aconcagua, $H$. canescens y $G$. blacodes estaría correlacionada directamente con la profundidad y densidad (sigma-t), e inversamente con la temperatura y salinidad. En cambio la abundancia relativa de $M$. gaudichaudi se asociaría directamente con la temperatura y salinidad e indirectamente con profundidad y densidad (sigma-t). Esta variable canónica reflejaría entonces las variaciones en profundidad de las condiciones ambientales a las cuales se asocian dichas especies (Tabla
Tabla 2. Correlación canónica, raíz característica $(\lambda)$, varianza explicada en porcentaje y valor $P$. $(*)$ indica la variable canónica significativa $(\mathbf{p}<\mathbf{0 , 0 5})$.

Table 2. Canonical correlation, characteristic $\operatorname{root}(\lambda)$, percent explained variance and corresponding $P$ value. $(*)$ indicate significant canonical variable $(\mathbf{p}<$ 0.05).

\begin{tabular}{|ccccc|}
\hline Vector & $\mathbf{R}$ & $\boldsymbol{\lambda}$ & Varianza $(\boldsymbol{\%})$ & Valor-P \\
\hline $1^{*}$ & 0,798 & 0,638 & 37,618 & 0,00 \\
$2 *$ & 0,649 & 0,421 & 24,823 & 0,01 \\
3 & 0,505 & 0,255 & 15,035 & 0,36 \\
4 & 0,432 & 0,186 & 10,966 & 0,67 \\
5 & 0,374 & 0,140 & 8,256 & 0,83 \\
6 & 0,237 & 0,056 & 3,302 & 0,94 \\
\hline
\end{tabular}

3). En el segundo vector, la especie Psammobatis sp. tuvo carga positiva y las variables salinidad y oxígeno disuelto tuvieron carga negativa y positiva respectivamente, dando relaciones directas con el oxígeno disuelto e indirectas con la salinidad ( $\mathrm{Ta}-$ bla 3$)$. 
Tabla 3. Cargas canónicas de los conjuntos de variables ambientales y biológicas correspondiente al invierno 1993. En negrita se indican los valores significativos.

Table 3. Canonical loadings of the environmental and biological variables for winter 1993. Significant values in bold.

\begin{tabular}{|lcc|}
\hline Variable & Vector $\mathbf{1}$ & Vector $\mathbf{2}$ \\
\hline Sustrato & $-0,421$ & 0,130 \\
Profundidad & $\mathbf{0 , 9 1 8}$ & $-0,302$ \\
Temperatura & $\mathbf{- 0 , 7 2 2}$ & $-0,028$ \\
Salinidad & $\mathbf{- 0 , 6 7 4}$ & $\mathbf{- 0 , 5 5 3}$ \\
Densidad & $\mathbf{0 , 6 4 3}$ & $-0,214$ \\
Oxígeno disuelto & 0,392 & $\mathbf{0 , 5 7 9}$ \\
Libidoclaea granaria & $\mathbf{0 , 5 7 7}$ & $-0,287$ \\
Merluccius gayi & 0,127 & 0,370 \\
Epigonus crassicaudus & $\mathbf{0 , 5 0 0}$ & $-0,253$ \\
Coelorhynchus aconcagua & $\mathbf{0 , 5 3 1}$ & $-0,056$ \\
Hippoglossina macrops & $-0,313$ & 0,138 \\
Mursia gaudichaudi & $\mathbf{- 0 , 5 5 6}$ & $-0,034$ \\
Centroscyllium nigrum & 0,318 & $-0,028$ \\
Halaelurus canescens & $\mathbf{0 , 5 7 1}$ & $-0,234$ \\
Raja chilensis & 0,407 & $-0,007$ \\
Genypterus maculatus & $-0,014$ & 0,400 \\
Prolatilus jugularis & $-0,253$ & $-0,018$ \\
Genypterus blacodes & $\mathbf{0 , 6 2 8}$ & 0,115 \\
Apristurus nasatus & 0,185 & 0,035 \\
Trachurus murphyi & $-0,141$ & $-0,144$ \\
Psammobatis sp. & 0,232 & $\mathbf{0 , 6 9 2}$ \\
Bramma australis & 0,094 & 0,222 \\
\hline
\end{tabular}

\section{DISCUSIÓN}

La estructura de las comunidades de peces y crustáceos en la zona centro-sur de Chile ha sido poco estudiada. En general, las investigaciones han estado orientadas principalmente a recursos de interés comercial (Yáñez et al., 1974; Yáñez, 1974; Yáñez $\&$ Barbieri, 1974), en los cuales se estudia la distribución y abundancia del recurso objetivo, más que el análisis de comunidades y su relación con las características oceanográficas. En este estudio, se detectó que los núcleos de alta diversidad se concentraron principalmente en el límite de la plataforma continental y talud superior, que son áreas donde ocurren los mayores cambios de la fauna, principalmente a lo largo del gradiente batimétrico (Overholtz \& Tyler, 1985; Fariña et al., 1997). En la zona batial, la presencia de estos núcleos de alta diversidad es inferior al 5\%. La plataforma continental estuvo dominada por un sustrato fangoso, mientras que el talud superior estuvo asociado a una mezcla de fango-arena-y roca, en tanto que la zona batial estuvo constituida por roca-laja. Ocho especies componen en un $90 \%$ estas zonas de alta diversidad: $C$. aconcagua, L. granaria, M. gaudichaudi, C. johni, C. porteri, C. nigrum, M. gayi e H. macrops. Los resultados de este trabajo concuerdan con Fariña $e t$ al. (1997), quienes determinaron que la organización de asociaciones de peces en diferentes áreas geográficas está determinada principalmente por la profundidad y estructura hidrográfica. Así, la mayor profundidad estaría asociada a un decrecimiento en densidad, biomasa y menor riqueza de especies.

La similitud faunística entre estaciones, basada en la abundancia relativa de las especies, determinó que 64 estaciones resultaron ser similares en un $80 \%$, cuya composición estuvo integrada por 35 especies. De estas especies, de acuerdo al ordenamiento de Fager, sólo 10 resultaron ser las más importantes: M. gayi, H. macrops, L. granaria, C. porteri, $M$. gaudichaudi, C. aconcagua, Pleuroncodes monodon, Raja chilensis, Genypterus maculatus y Epigonus crassicaudus. Según su distribución batimétrica, estas especies se localizaron mayoritariamente sobre la plataforma. No obstante, $G$. maculatus, L. granaria, C. porteri, P. monodon y $R$. chilensis, se localizaron de preferencia en la plataforma y talud continental en el rango 101-350 m; $E$. crassicaudus se ubicó en el talud superior (201-350 m); y M. gayi (especie dominante del sistema), $H$. macrops, $M$. gaudichaudi y $C$. aconcagua presentaron una distribución más amplia en profundidad $(101$ a $550 \mathrm{~m})$. Estas especies estuvieron asociadas a más de un tipo de sustrato.

Cada asociación de especies estaba dominada únicamente por unas pocas especies, por lo tanto, el número total de especies fue relativamente bajo, coincidiendo con los resultados de Arancibia (1992), quien encontró que $M$. gayi es más importante alejada de la costa y se encuentra asociada con $H$. macrops. Sin embargo, excluyendo el rango de 50 a $100 \mathrm{~m}$ de profundidad que no fue observado en este estudio, M. gayi siempre fue la especie dominante incluso en el talud, aún en presencia de $C$. aconcagua, sin que se evidenciara un eventual reemplazo de la especie como lo señala Arancibia (1992). 
Los resultados de este trabajo indican que las asociaciones de la fauna íctica y de crustáceos bentodemersales en la zona centro-sur de Chile, exhiben tres asociaciones de especies, las cuales presentan una clara segregación en profundidad. La asociación entre M. gayi, $R$. chilensis, G. blacodes, C. aconcagua, E. crassicaudus y L. granaria, con dos especies asociadas: D. calcea y $C$. fasciatus se distribuye presentando una amplia distribución batimétrica $(101-550 \mathrm{~m})$, entre $33^{\circ}$ y $36^{\circ} \mathrm{S}$, ubicándose de preferencia en la zona del talud superior (67\% de las veces), asociado a dos tipos de sustratos, fango y roca-laja.

El grupo recurrente caracterizado por la presencia de $H$. macrops, C. nigrum, C. porteri, $M$. gaudichaudi y $H$. canescens, restringido al límite de la plataforma continental en los $36^{\circ} \mathrm{S}$, se asocia al sustrato fango-arena-roca. En la zona batial se presenta una asociación constituida por Bathyraja sp., M. magellanicus, G. maculatus y $S$. oculatus. Esta asociación se presenta sobre un sustrato de características duras (roca-laja) en los $36^{\circ} \mathrm{S}$.

Se determinó también que los grupos recurrentes se asocian con el AESS y a la mezcla entre la AESS y el Agua Intermedia Antártica (AIAA), esto se podría explicar debido a que el crucero estuvo dirigido a la evaluación específica de un recurso, la merluza común $M$. gayi, que se asocia preferentemente a la AESS. Estas asociaciones de los grupos recurrentes con las masas de agua puede observarse en la figura 3. La asociación del grupo recurrente A, tiende a ubicarse en una mezcla entre la AESS y
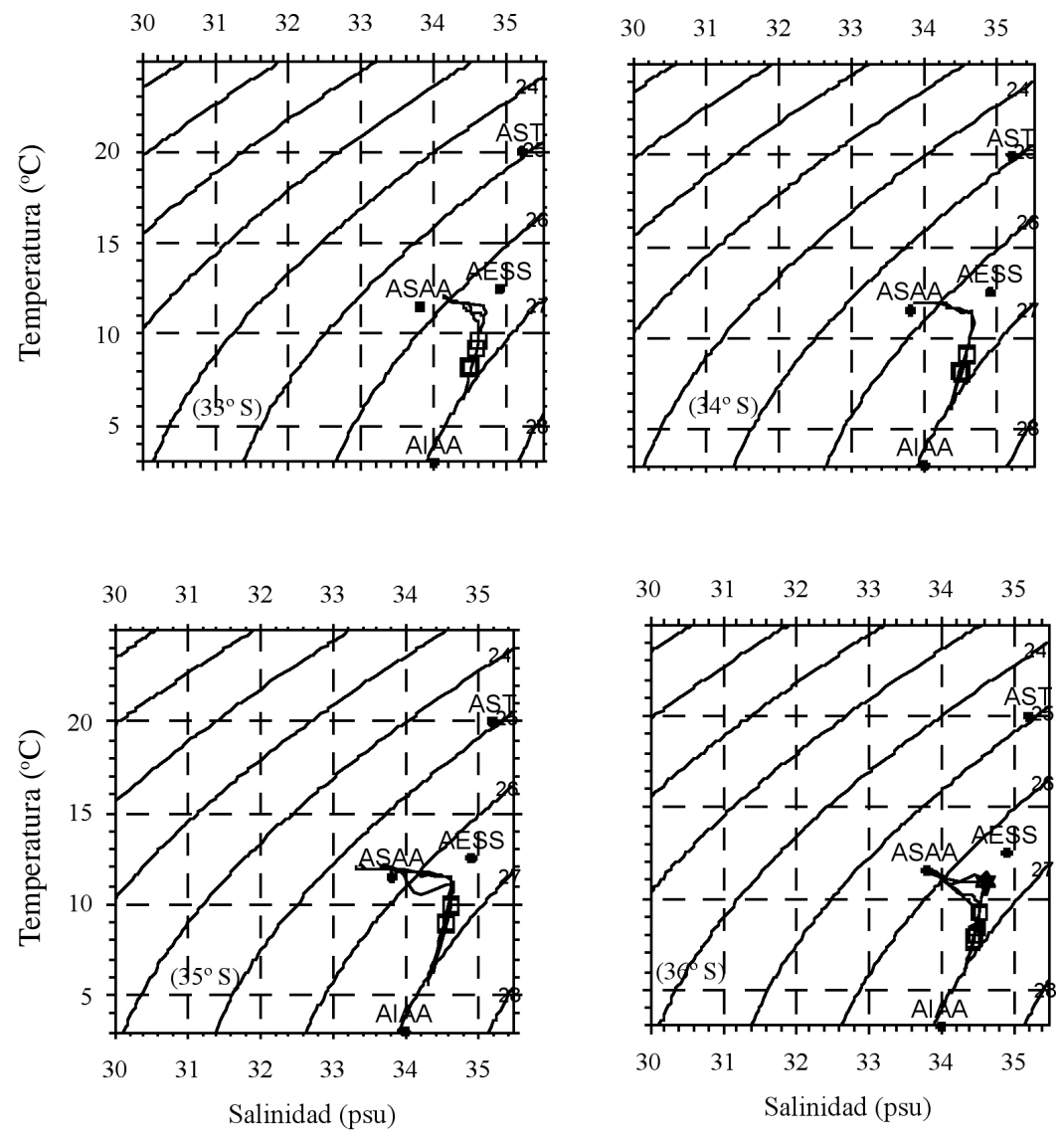

Grupo recurrente A

Grupo recurrente B

Grupo recurrente $\mathrm{C}$

Figura 3. Diagramas T-S mostrando la ubicación de los grupos recurrentes A, B y C en invierno de 1993, entre $33^{\circ}$ y $36^{\circ} \mathrm{S}$. $\square$ : Grupo recurrente $A, \star$ : Grupo recurrente $B, \angle$ : Grupo recurrente $C$.

Figure 3. T-S diagrams showing the distribution of the recurrent groups A, B, and C during winter 1993, between

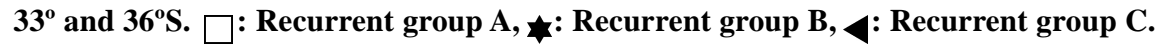


AIAA. De acuerdo a Fedele (1993), a esa latitud y en los estratos batimétricos 201-350 y 351-550 m, el AESS está presente entre un 40-50\%. La asociación del grupo recurrente B presente en los $36^{\circ} \mathrm{S}$ a profundidades entre 101 y $200 \mathrm{~m}$, se ubica en el núcleo del AESS, a esa latitud y a esa profundidad el AESS está presente en un 40\%. La asociación del grupo recurrente $\mathrm{C}$, en el rango batimétrico 201-350 m estuvo asociado a la mezcla de la AESS y AIAA.

Yáñez (1978), encontró una marcada discontinuidad entre la fauna de la plataforma y la zona batial, situación que se refleja también en este caso. En cada zona la composición faunística varió gradualmente con la profundidad. Así, en la plataforma continental se encuentran especies estrictamente costeras y especies distribuidas sobre toda la plataforma (hasta los $220 \mathrm{~m}$ ). En cambio, en la zona batial, se distinguen las especies estrictamente profundas (distribuidas más allá de los $400 \mathrm{~m}$ ), de las especies repartidas entre los 300 y $600 \mathrm{~m}$, y de las especies repartidas en toda la zona, M. gayi, $G$. maculatus y $R$. chilensis.

Del análisis de correlación canónica, para el invierno de 1993, se deduce que los focos de abundancia relativa de L. granaria, E. crassicaudus, $C$. aconcagua, $H$. canescens y $G$. blacodes tienden a asociarse directamente con la profundidad y densidad, e indirectamente con la temperatura y salinidad. La asociación de $G$. blacodes con la profundidad se confirma por la distribución batimétrica de esta especie, entre los 50 y $500 \mathrm{~m}$ de profundidad y su concentración entre 200 y 400 m (Avilés et al., 1979). Esta asociación podría estar vinculada con sus hábitos tróficos ya que preda sobre peces $(C$. fasciatus, M. gayi) y crustáceos $(H$. reedi, $P$. armata, $P$. monodon), cuyas abundancias se distribuyen en estos mismos rangos batimétricos.

La abundancia de Psammobatis sp. se correlaciona directamente con la concentración de oxígeno disuelto, es decir, al incrementar la concentración de oxígeno, la abundancia relativa de Psammobatis sp. tiende aumentar.

En conclusión, se puede señalar que la segregación aparente de los grupos responde en parte a la batimetría, pero la heterogeneidad en la naturaleza y distribución del sustrato en el área de estudio, es un factor que influye en la composición de los grupos. Sin embargo, sería importante considerar el grado de permanencia de los grupos recurrentes en el tiempo e investigar las relaciones tróficas de sus componentes sobre la base de que existe una rela- ción tipo depredador-presa, que eventualmente sería más determinante en la consistencia de las asociaciones encontradas.

\section{AGRADECIMIENTOS}

Los autores agradecen al Instituto de Fomento Pesquero (IFOP) y al Fondo de Investigación Pesquera (FIP), por su autorización para el uso de la información obtenida en el crucero de investigación "Evaluación directa de hidroacústica del stock de merluza común en la zona centro-sur de Chile".

\section{REFERENCIAS}

Aguayo, M. \& H. Robotham. 1984. Dinámica poblacional de merluza común (Merluccius gayi gayi). Invest. Pesq., Santiago, 31: 17-45.

Arana, P., T. Melo, L. Noziglia, I. Sepúlveda, N. Silva, G. Yany \& E. Yáñez. 1975. Los recursos demersales de la región de Valparaíso-Chile. Com. Perm. Pacífico Sur, 3: 39-6 1.

Arancibia, H. 1991. Análisis ecológico pesquero del recurso langostino colorado (Pleuroncodes monodon) y su interacción con merluza común (Merluccius gayi) y lenguado de ojos grandes (Hippoglossina macrops). Rev. Biol. Mar., Valparaíso, 20: 37-48.

Arancibia, H. 1992. Patrones de distribución de la asociación de peces demersales frente a Chile central. Rev. Biol. Pesq., 21: 43-53.

Burukovskii, R. 1992. Key to shrimps and lobsters. Pishchevaya Promyshlennost Publishers, Moscow, $174 \mathrm{pp}$.

Cañón, J. \& E. Morales. 1985. Geografía de Chile. IX Geografía del mar chileno. Instituto Geográfico Militar, Santiago, 244 pp.

Céspedes, R., L. Adasme, C. Vera \& H. Miranda. 2001. Investigación estado de situación pesquería demersal zona sur-austral. Programa de seguimiento del estado de situación de las principales pesquerías nacionales. Informe Final, IFOP, Chile, 60 pp.

Fager, E.W. 1957. Determination and analyses of recurrent groups. Ecology, 38: 586-595. 
Fager, E.W. \& J.A. McGowan. 1963. Zooplankton species groups in the North Pacific. Science, 140: 453-460.

Fariña, A.C., J. Freire \& E. González-Gurriarán. 1997. Demersal fish assemblages in the Galician continental shelf and upper slope (NW Spain): Spatial structure and long-term changes. Estuar. Coast. Shelf Sci., 44: 435-454.

Fedele, A. 1993. Razón $\mathrm{N}-\mathrm{NO}_{3}: \mathrm{P}_{-}-\mathrm{PO}_{4}$ anómala, un trazador químico para la masa de Agua Ecuatorial Subsuperficial (AESS). Tesis de Oceanografía. Universidad Católica de Valparaíso, 134 pp.

Lalli, C. \& T. Parsons. 1993. Biological oceanography an introduction. Butterworth-Heinemann, Oxford, 314 pp.

Manly, B. 1994. Multivariate statistical methods. Chapman \& Hall, London, 215 pp.

Ojeda, P. 1983. Distribución latitudinal y batimétrica de la ictiofauna demersal del extremo austral de Chile. Rev. Chil. Hist. Nat., 56: 61-70.

Overholtz, W. \& A. Tyler. 1985. Long-term responses of the demersal fish assemblages of Georges Bank. US Fish. Bull., 83(4): 507-520.

Palma, S., R. Ulloa, L. Linacre \& P. Arana. 1998. Estudio de los hábitos alimentarios de la merluza común Merluccius gayi gayi (Guichenot, 1948), frente a Papudo (32³0’S) y su relación con las condiciones oceanográficas. Estud. Doc., Univ. Católica de Valparaíso, 10/98, 48 pp.

Pohle, G., F. Montelatto \& M.L. Negreros-Frangozo. 1999. Larval Decapoda (Brachuyra). En: D. Boltovskoy (ed.). South Atlantic Zooplankton. Backhuys Publishers, Leiden, pp. 1281-1351.

Pielou, E.C. 1977. Mathematical ecology. John Wiley \& Sons, New York, 384 pp.

Retamal, M.A. 1981. Catálogo ilustrado de los crustáceos Decápodos de Chile. Gayana, zool., 44: 1-110.

Retamal, M.A. 1994. Los decápodos de Chile. Facultad de Ciencias Biológicas, Universidad de Concepción, 256 pp.

Rocha, G. \& C. Rossi-Wongtschowski. 1998. Demersal fish community on the inner shelf of Ubatuba, southeastern Brazil. Rev. Bras. Oceanogr., 46(2): 93-109.

Recibido: 4 junio 2004; Aceptado: 18 abril 2005
Sanders, H.L. 1960. Benthic studies in Buzzards Bay. III. The structure of the soft-bottom community. Limnol. Oceanogr., 5: 138-153.

Shannon, C.E. \& W. Weaver. 1949. The mathematical theory of communication. University of Illinois Press, Urbana, 117 pp.

Servicio Hidrográfico y Oceanográfico de la Armada (SHOA). 1981. Carta Pesquera CP 700. Bahía Corral a isla Guafo.

Servicio Hidrográfico y Oceanográfico de la Armada (SHOA). 1983. Carta Pesquera CP 600. Golfo de Arauco a bahía Corral.

Servicio Hidrográfico y Oceanográfico de la Armada (SHOA). 1984. Carta Pesquera CP 400. Coquimbo a Valparaíso.

Vidal, R., E. Aranda, Z. Young, M. Aguayo \& S. Chomalí. 1985. Perfiles indicativos del sector pesquero nacional, recursos, tecnologías y mercado. Santiago, Chile, IFOP (AP 85/12), 131 pp.

Wisner, R. 1974. The taxonomy and distribution of lanternfishes (Family Myctophidae) of the Eastern Pacific Ocean. Navy Ocean Research and Development Activity, Mississippi, 229 pp.

Yáñez, E. 1974. Distribución y abundancia relativa estacional de los recursos disponibles a un arte de arrastre camaronero frente a la costa de Valparaíso. Invest. Mar., Valparaíso, 5(5): 125-135.

Yáñez, E. 1978. Etude de la répartition par des analices d'inertie et estimation de la biomasse saisonniere de la faune demersale de la région de Valparaiso, Chili $\left(33^{\circ} \mathrm{S}, 72^{\circ} \mathrm{W}\right)$. Cah. ORSTOM, sér. Océanogr., 16(3-4): 319-348.

Yáñez, E. \& M.A. Barbieri. 1974. Distribución y abundancia relativa de los recursos disponibles a un arte de arrastre camaronero frente a la costa de Valparaíso. Invest. Mar., Valparaíso, 5(6): 137-156.

Yáñez, E., H. Trujillo, M.A. Barbieri \& T. Melo. 1974. Distribución y abundancia relativa estacional de los recursos disponibles a un arte de arrastre merlucero frente a la costa de Valparaíso. Invest. Mar., Valparaíso, 5(4): 111-125.

Zamora, C. 1991. Catálogo descriptivo de las principales especies de peces, crustáceos y moluscos, comunes en la región de Aysén, Chile. Inf. Tec. Pesca Chile, 41: 1-145. 